\section{Enteric fever cases showing concurrent seropositivity with Dengue and malaria: A sero-diagnostic challenge}

\author{
Ramya T.G., ${ }^{1}$ Sunitha B.R. ${ }^{2}$ \\ ${ }^{1}$ Department of Microbiology, Karuna \\ Medical College, Vilayodi, Palakkad, \\ Kerala; ${ }^{2}$ Department of Microbiology, \\ JJM Medical College, Davangere, \\ Karnataka, India
}

\begin{abstract}
Enteric fever, Dengue and malaria still remain diseases of public health importance in the tropics. Individuals residing in endemic areas are at risk of contracting these infections either concurrently or an acute infection superimposed on a chronic one. This study was undertaken to document patients showing co seropositivity for Enteric fever, Malaria and Dengue and to record the baseline Salmonella antibody titer in voluntary blood donors who represent general population of the area. The present study was conducted in the Department of Microbiology, J.J.M. Medical College, Davangere. Among the 824 febrile patients enrolled with positive serological test for either Typhoid, Dengue or Malaria, 189 patients were found to have co seropositivity to any two of the above diseases on subjecting their serum to Widal test, Dengue ELISA and malaria antigen detection by immunochromatography. A total of 189 patients showed co seropositivity for any of the above mentioned diseases accounting for $22.90 \%$. Typhoid-Dengue was found in $9.83 \%$ Typhoid-Malaria in IgG antibody level and sharing of antigen and cross reacting antibodies.

Poor diagnosis continues to hinder Malaria, Typhoid and Dengue control in the tropics. This is due to a combination of factors including nonspecific clinical presentation of the diseases, high prevalence of asymptomatic infection in many areas, lack of resources of insufficient access to train health care providers and health facilities, widespread practice of self treatment for clinically suspected malaria and typhoid fever. $^{2}$

Baseline titer of Salmonella antibodies for interpreting significant/diagnostic titer are not always available in every region and remain unrevised for decades together.

To overcome these difficulties, tests employing rapid antigen detection, detection of post infection IgM antibodies and review of baseline titers for laboratory diagnosis can serve as alternatives. Seropositivity for more than one of the above tests makes both laboratory and clinical diagnosis difficult. Hence this study was done to assess the frequency of concurrent infections with Dengue and Malaria in Enteric fever patients in Davangere.
\end{abstract} $6.67 \%$ and all the three in $0.48 \%$. DengueMalaria co-seropositivity was recorded in $5.94 \%$. The basal titer in healthy population was found to be $<1: 20$. The co seropositivity rate in our study is $22.9 \%$ which poses a challenge in the diagnosis and treatment of such patients. As the gold standards culture and microscopy are time consuming and molecular diagnostic tools not a practical reality in many rural and developing primary health centers, simple, rapid and sensitive serological methods are being used as an alternative diagnostic tool in diagnosing atypical co infections which in some instance leads to overwhelming diagnosis of co infections and improper treatment.

\section{Introduction}

Typhoid, Malaria and Dengue fever are among the most endemic diseases in the tropics. Though caused by different agents, they have similar clinical presentation. Etiological diagnosis is important in the management of these diseases which are associated with population density, urbanization, endemicity and mobility all favoring the disease spread. ${ }^{1}$

With the availability of rapid serodiagnostic tests for these infections, it has been observed that patients' samples frequently show seropositivity for two or more infections posing challenges in clinical diagnosis and treatment. The reasons could be endemicity of the disease leading to raised

\section{Materials and Methods}

\section{Type of study}

The present study was a type of cross sectional study conducted in the department of Microbiology, JJM Medical College, Davangere from July 2010 to June 2012.

\section{Source of data}

Febrile patients attending the outpatient department or admitted to hospitals attached to JJM Medical College suspected to have signs and symptoms clinically suggestive of typhoid, malaria and/or dengue were initially enrolled. Informed consent was taken from all patients during the study.

\section{Method for collection of data \\ Inclusion criteria: Febrile patients with}

Correspondence: Ramya T.G., Department of Microbiology, Karuna Medical College, Vilayodi, Chittur, Palakkad, Kerala, 678103, India

Tel.: +9946448439.

E-mail: tgramya@yahoo.com

Key words: Co-seropositivity; Widal; ELISA; Immunochromatography.

Acknowledgements: the authors would thank Dr. Vishwanath.G. HOD, Dept of Microbiology, JJMMC, Davangere for all the support during the conduct of the study.

Contributions: the authors contributed equally

Conflict of interest: the authors declare no potential conflict of interest.

Received for publication: 20 July 2017.

Revision received: 30 July 2017.

Accepted for publication: 14 November 2017.

This work is licensed under a Creative Commons Attribution NonCommercial 4.0 License (CC BY-NC 4.0).

CC Copyright R.T.G. and S.B.R., 2017

Licensee PAGEPress, Italy

Microbiology Research 2017; 8:5564

doi:10.4081/mr.2017.5564

positive serological tests for either Typhoid, Malaria or Dengue were included.

Exclusion criteria: Febrile patients showing negative results in serological tests for Typhoid, Malaria and Dengue and/or seropositive for one of the above infections, TAB vaccinated cases and fever due to other proven causes were excluded.

Sample size: A) 100 voluntary blood donors for determining baseline titers; B) 189 cases showing concurrent seropositivity for Dengue, Malaria and/or Typhoid.

\section{Specimen collection}

Using strict aseptic precautions, $5-8 \mathrm{~mL}$ of venous blood was collected in appropriate sterile bulbs. $2 / 3$ of the blood was allowed to clot at room temperature for half an hour, after which the clot was dislodged to separate the serum. This was centrifuged at $3000 \mathrm{rpm}$ for 2 minutes. This was used for Dengue IgM and IgG ELISA and Widal test and for detection of S.typhi IgM antibodies using immunochromatography (Enterocheck-WB)

Remaining $1 / 3$ blood was added to the EDTA bulb and this anticoagulated blood was subjected to Malarial antigen detection using rapid immunochromatography (Malarigen kit). 
regions is largely unknown and is yet a field to be explored in India. No significant data about such an association is available in India. With the advent of rapid diagnostic serological tests for diagnosis of these diseases, there have been a lot of challenges in laboratory and clinical diagnosis of coinfection. This has prompted us to undertake the present study. Widal test, Dengue IgM/IgG ELISA and Malaria rapid antigen immunochromatography were used to screen Typhoid, Dengue and Malaria cases.

Although blood culture is the confirmatory test for diagnosing Typhoid, in many developing areas, still patients are not able to afford the cost factor. The patients are invariably treated with empirical antibiotics even before a culture report is sought. The baseline titer of Salmonella antibodies needs revision from area to area over a period of time. Hence in this study, 100 serum samples from healthy volunteers (blood donors) were considered for baseline Salmonella antibody titer estimation.

In the present study, $6.0 \%$ of the serum samples showed a significant titer of anti-O antibodies $(\geq 1: 80)$ and $11.0 \%$ showed a significant titer of anti-H antibodies $(\geq 1: 80)$.

The results are comparable with the study conducted by Shukla et al. ${ }^{3}(13.83 \%$ and $8 \%)$, Jeyakumari et $a .^{4}(2.2 \%$ and $4,4 \%)$ and Peshattiwar $^{5}$ (4.1\% and 9.52\%). Slightly higher value for $\mathrm{H}$ antibodies $(29.0 \%)$ was recorded by Pokhrel et al. ${ }^{6}$ in Nepal.

Majority of the blood donors had a titer of $\leq 1: 20$ for both $\mathrm{O}$ and $\mathrm{H}$ antibodies. The reason for the low titer could be better awareness among the population and improved social hygiene. This warrants future studies on baseline tires on a larger population and periodic review of the baseline titers in this geographic location.

A total of 55 patients showed co seropositivity for typhoid and malaria accounting for $6.67 \%$. This is slightly lower compared to the findings of Mbuh et al. ${ }^{7}$ $(10.1 \%)$, Olopoenia et al. ${ }^{8}(12 \%)$, Jhaveri et $a l .{ }^{9}(14.58 \%)$ and Samal and Sahu ${ }^{10}$ (15.4\%). A slightly higher percentage was observed by Igharo et al. in Nigeria. ${ }^{11}$

Detection of Malaria parasite in a peripheral blood smear by conventional light microscopy remains currently the gold standard for diagnosis of Malaria. It can be used for speciation. It is sensitive, informa- tive, relatively inexpensive. It is a general diagnostic technique that can be shared with other disease control programs and can provide a permanent record (the smears) of the diagnostic findings and be subject to quality control. ${ }^{12}$ Recently, there is gradual replacement of microscopy by rapid immunochromatographic tests for detection of Malarial antigen which can be done and interpreted by inexperienced personnel in a relatively shorter time.

The definitive diagnosis of Typhoid fever requires the isolation of Salmonella enterica serotype typhi from the patient. However, this requires laboratory equipment and technical training that are beyond the means of most primary health care facilities in the developing world. Consequently, Widal test is the only specific diagnostic investigation available in most tropical regions. ${ }^{13} \mathrm{~A}$ fourfold rise in titer of antibodies was not detected as patients were not available for repeat sample.

It has been shown that antibody response to $\mathrm{O}$ antigen of S.typhi was markedly reduced in acute episode of Malaria compared with that in controls where humoral immunity is transiently impaired. ${ }^{14}$

As the typhi antigen is shared by a large number of organisms from the Salmonella genus and other related organisms, ${ }^{15,16}$ a positive Widal test is therefore likely to occur in several conditions other than the actual Salmonella enteric infection. False positive Widal test has already been reported in other febrile illnesses such as Malaria, Tuberculosis, and Schistosomiasis. ${ }^{17-19}$

The outcome of the Widal reaction for patients with a clinical suspicion of Typhoid and Malaria depends on individual host immune responses, which become stimulat-

Table 1. Baseline Salmonella antibody titers in healthy volunteers (blood donors).

\begin{tabular}{lcccc} 
Titer & TO & TH & AH & BH \\
$<1: 20$ & 58 & 48 & 97 & 100 \\
$1: 20$ & 14 & 17 & - & - \\
\hline $1: 40$ & 22 & 24 & - & - \\
$1: 80$ & 01 & 05 & - & - \\
\hline $1: 160$ & 03 & 04 & 01 & - \\
$1: 320$ & 02 & 02 & 02 & - \\
\hline Total & 100 & 100 & 100 & 100 \\
\hline & & & &
\end{tabular}

Table 2. Seropositivity of typhoid-dengue.

\begin{tabular}{|c|c|c|c|c|c|c|c|c|}
\hline \multirow{2}{*}{ Total cases } & \multicolumn{2}{|c|}{ Positive } & \multicolumn{2}{|c|}{ Dengue IgM + typhoid } & \multicolumn{2}{|c|}{ Dengue IgG + typhoid } & \multicolumn{2}{|c|}{ Dengue IgM + IgG + typhoid } \\
\hline & N. & $\%$ & N. & $\%$ & N. & $\%$ & N. & $\%$ \\
\hline 824 & 81 & 9.83 & 46 & 56.79 & 26 & 32.09 & 9 & 11.11 \\
\hline
\end{tabular}


Table 3. Seroprevalence of dengue and malaria co seropositivity.

\begin{tabular}{lcc} 
Suspected cases & Dengue-malaria co-infection & Percentage \\
824 & 49 & $5.94 \%$ \\
\hline
\end{tabular}

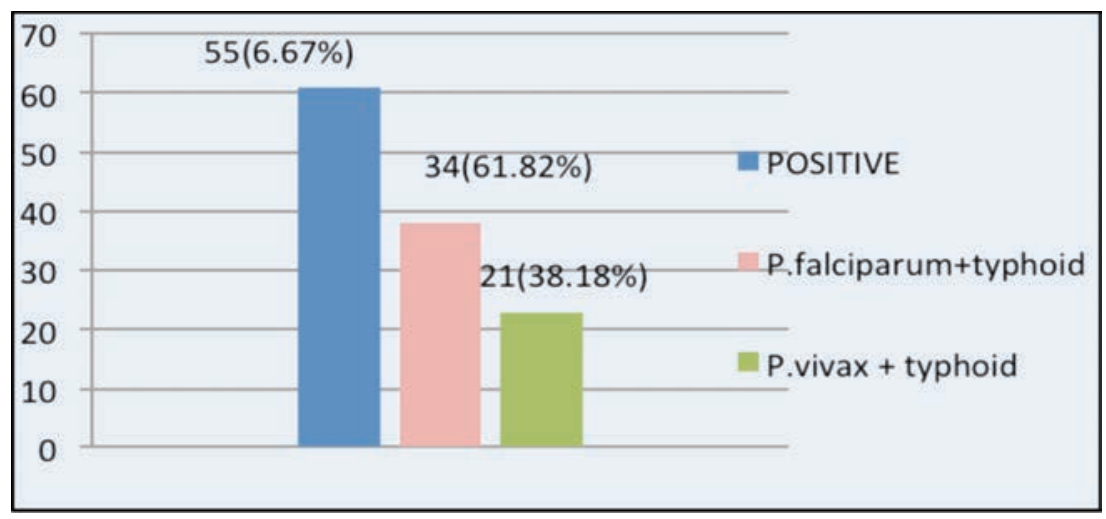

Figure 1. Seropositivity of typhoid-malaria.

ed in febrile conditions associated with Malaria fever. The memory response could cause positive Widal reactions in previously sensitized patients. This could be a reason for false positive Widal test. ${ }^{20}$

There are only a couple of reports available on co-infection with Dengue and Typhoid.

In the present study, we recorded 81 patients showing co seropositivity for Dengue and Typhoid accounting for $9.83 \%$. $56.79 \%$ had $\operatorname{IgM}, 32.09 \%$ had $\operatorname{IgG}$ and $11.11 \%$ had both IgM and IgG. In secondary Dengue there is a high titer of IgG and a low titer of IgM. This could be the reason for the presence of both $\operatorname{IgG}$ and $\operatorname{IgM}$ in 09 patients. The actual reason for concurrent Dengue Typhoid co infection remains unclear. The possibility could be either a camouflaging of symptoms in a true co infection or it could be a superimposed secondary infection. The definitive diagnosis can be established only by culture.

All the 140 Widal test seropositive cases were subjected to IgM immunochromatography. The results of the present study show a lower value than those of Anusha et al. ${ }^{21}$ (47\%) and Anagha et al. ${ }^{22}$ (20.48\%). We compared Enterocheck with Widal test and found the sensitivity to be very low (5.07\%). The above authors have considered blood culture instead of Widal test. Our findings suggest that Enterocheck is not very suitable for diagnosing enteric fever.

\section{Conclusions}

Understanding the nature and conse- quences of co-infection is vital for accurate estimates of infectious disease burden. Improved knowledge of the factors controlling an individual's risk of co-infection and the mechanisms behind these pathogenpathogen interactions, especially from experimental studies, will also aid the proper management of these co-infections. Relying only on concurrent seropositivity of one or more tests leads to a overwhelming diagnosis of co-infection. A serological test should always be confirmed with a gold standard method of diagnosis to avoid improper diagnosis and treatment.

\section{References}

1. Butel JS. Arthropod-borne and rodent borne viral diseases. In: Brooks GF, Carroll KC, Butel JS, et al (eds). Melnick and Adelberg's medical microbiology. 24th ed. New York: McGraw Hill; 2007. pp 725-729.

2. Igbenehu $\mathrm{C}$, Olisekodiaka MJ, Onuegbu JA. Malaria and typhoid fever among adult patients presenting with fever in Ibadan, Southwest Nigeria. Int J Trop Med 2009;52:200-2.

3. Shukla S, Patel B, Chitnis DS. 100 years of Widal \& its reappraisal in an endemic area. Indian J Med Res 1997;105:53-7.

4. Sneha AJ. Study of the baseline Widal titres among healthy individuals in Puducherry; 2011. Available from: http://smvmch.ac.in/sites/default/files/S tudyofthe baselineWidal titres among healthy.pdf.
5. Peshattiwar P. Study of the baseline widal titre amongst healthy individuals in Amlapuram, India. J Clin Diagnost Res 2012;6:416-7.

6. Pokhrel BM, Karmacharya R, Mishra SK, Koirala J. Distribution of antibody titer against Salmonella enterica among healthy individuals in Nepal. Ann Clin Microbiol Antimicrob 2009;8.

7. Mbuh FA, Galadima M, Ogbadu L. Rate of coinfection with malaria parasites and Salmonella typhi in Zaria, Kaduna state, Nigeria. Ann Afr Med 2003;2:64-7.

8. Olopoenia L, Oyewole F, Onafowokan R1. Widal agglutination in malaria infection. Med Rev 1996;3:5-6.

9. Jhaveri KN, Nandwani SK, Mehta PK, et al. False positive modified Widal test in acute malaria. J Assoc Physicians India 1995;43:754-5.

10. Samal KK, Sahu JCS. Malaria and Widal reaction. J Assoc Physicians India 1991;39:745-7.

11. Igharo EA, Osazuwa F, Ajayi SA, et al. Dual Infection with Typhoid and Malaria in Febrile Patients in Ikare Akoko, Nigeria. Int J Trop Med 2012; 7:49-52.

12. World Health Organization. Malaria diagnosis new perspectives: report of a joint WHO/USAID informal consultation. Geneva: World Health Organization: 2000. pp 1-57.

13. Uneke CJ. Concurrent malaria and typhoid fever in the tropics: the diagnostic challenges and public health implications. J Vector Borne Dis June 2008;45:133-42.

14. Khan MA, Mekan SF, Abbas Z, Smego RA Jr. Concurrent malaria and enteric fever in Pakistan. Singapore Med J 2005;46:635-8.

15. Chart H, Waghorn DJ, Rowe B. Serological detection of patients infected with Salmonella enteritidis. Serodiagn Immunother Infect Dis 1994;6:79-81.

16. Wang W, Perepelov AV, Feng L, et al. A group of Escherichia coli and Salmonella enterica $\mathrm{O}$ antigens sharing a common backbone structure. Microbiology 2007;153:2

17. Ghosh K, Javeri KN, Mohanty D, et al. False positive serological tests in acute malaria. Br J Biomed Sci 2001;58:20-3.

18. Senewiraine B, Senewiratne K. Reassessment of the widal test in the diagnosis of typhoid. Gastroenterology 1977;73:233-6.

19. Skoutelis A, Dimitrakopoulos G, Bassaris H. False positive widal reaction in high-titer disseminated BCG infection. Eur J Clin Microbiol Infect 
Dis $1994 ; 13: 261-3$.

20. Mbuh FA, Galadima M, Ogbadu L. Rate of co infection with malaria parasites and Salmonella typhi in Zaria, Kaduna state, Nigeria. Ann Afr Med 2003;2:64-7.
21. Anusha R, Ganesh R, Lalitha J. 22. Anagha K, Deepika B, Shahriar R, Comparison of a rapid commercial test, Enterocheck WB® with automated blood culture for diagnosis of typhoid fever. Ann Trop Int Child Health 2011;31;231-4. Sanjeev K. The easy and early diagnosis of typhoid fever. J Clin Diagn Res 2012;6:198-9. 